\title{
Manejo anestésico en la enfermedad de Steinert. A propósito de un caso
}

\author{
Anesthetic management in Steinert's disease: case report \\ Dra. Yaiza Beatriz Molero-Díez,* Dr. Víctor Javier Sánchez-Hernando,* \\ Dr. Francisco Antonio Ruiz-Simón,* Dra. María Gómez-Fernández,* \\ Dra. María Teresa Mateos-Arribas,* Dr. Francisco García-Lázaro*
}

Citar como: Molero-Díez YB, Sánchez-Hernando VJ, Ruiz-Simón FA, Gómez-Fernández M, Mateos-Arribas MT, García-Lázaro F. Manejo anestésico en la enfermedad de Steinert. A propósito de un caso. Rev Mex Anest. 2021; 44 (1): 66-69. https://dx.doi.org/10.35366/97780

\begin{abstract}
RESUMEN. Se presenta el caso de paciente masculino, de 60 años, programado para resección transuretral de próstata. Como antecedentes destacan enfermedad de Steinert e implantación de marcapasos. La enfermedad de Steinert es el antecedente principal que guiará nuestra práctica anestésica y, tras valorar el tipo de intervención prevista, se decide anestesia locorregional, dadas las potenciales complicaciones que pueden presentar estos pacientes con la anestesia general. La conducta anestésica de los pacientes con enfermedad de Steinert supone un reto para el anestesiólogo tanto por la gran cantidad de complicaciones que pueden aparecer en el intra- y en el postoperatorio, como por la baja frecuencia de esta enfermedad. Además, el estrés quirúrgico y las técnicas utilizadas pueden interferir en el curso de la enfermedad. Por todo ello, el abordaje y los cuidados intra- y postoperatorios se deben planificar y seleccionar con cuidado con el fin de obtener los mejores resultados y extremar la seguridad del paciente.
\end{abstract}

ABSTRACT. A 60-year-old man with prostatic hypertrophy was scheduled for transurethral resection of the prostate. Steinert's disease and implantation of a pacemaker were his previous pathology. Being Steinert's disease the most relevant clinical characteristic and the type of intervention urologist has planned, we decide locoregional anesthesia technique, avoiding the potential complications that these patients may present with general anesthesia. The anesthetic management of Steinert's disease patients is a challenge for the anesthesiologist both due to the large number of complications that may appear during intra- and postoperative time as well as the low frequency of this pathology. In addition, surgical stress and the techniques we use can interfere with the course of the disease. Therefore, the approach and immediate intra-and postoperative care should be carefully planned and selected in order to obtain the best results and maximize patient safety.

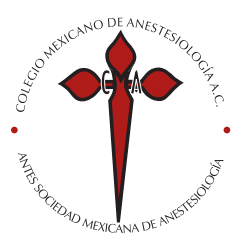

Palabras clave:

Anestesia endotraqueal, anestesia raquídea, bloqueo neuromuscular, distrofia miotónica, hiperplasia prostática.

Keywords:

Anesthesia endotracheal, anesthesia spinal, benign prostatic hyperplasia, myotonic dystrophy, neuromuscular blockade.

\footnotetext{
* Departamento de Anestesiología y Reanimación del Hospital Virgen de la Concha. Zamora, España.
}

\section{Correspondencia:}

Yaiza Beatriz Molero-Díez Departamento de Anestesiología

y Reanimación del Hospital

Virgen de la Concha.

Av. Requejo Núm. 35, 49004, Zamora (España).

Teléfono: 980548210

Fax: 980512838

E-mail: yaiza923@hotmail.com

Recibido para publicación: 27-09-2019

Aceptado para publicación: 14-11-2019

\section{INTRODUCCIÓN}

$\mathrm{L}$ a enfermedad de Steinert, también llamada distrofia miotónica tipo I, es la miopatía más prevalente en el paciente adulto con una incidencia de tres a cinco pacientes por cada 100,000 personas $^{(1)}$.

Su transmisión sigue una herencia autosómica dominante, con penetrancia completa y una expresividad variable producida por la expansión de tripletes CTG en la región no codificante del gen DMPK (proteincinasa de la distrofia miotónica), localizado en el cromosoma $19 q^{(2)}$. El inicio de esta enfermedad suele ser entre la segunda y la tercera década de la vida ${ }^{(3)}$.

Es una enfermedad sistémica con alteración de prácticamente todos los órganos y tejidos. A pesar de ello, se trata fundamentalmente de una enfermedad del músculo que ocurre por un defecto de la función de los canales $\mathrm{Na}-\mathrm{Cl}$ que ocasiona inestabilidad eléctrica de la membrana muscular y con ello una despolarización mantenida de la misma y ocasiona la sintomatología más característica de la enfermedad: la miotonía y la atrofia muscular.

Las principales manifestaciones clínicas ${ }^{(3-6)}$ son:

\section{Neurológicas:}

1) Miotonía: contracción persistente no dolorosa tras una contracción voluntaria.

2) Debilidad muscular: de predominio fundamentalmente distal, afectándose también los músculos de la cara, deglución y fonación.

3) Hipertermia maligna. 
4) Fatiga e hipersomnia.

5) Retraso intelectual y alteraciones de la personalidad.

- Cardíacas: se estima en torno a 75-80\% de los pacientes, con espectro clínico variable, desde alteraciones leves hasta arritmias graves que pueden ocasionar muerte súbita:

1) Trastorno del ritmo: se afecta el sistema de conducción, como bloqueos auriculoventriculares (AV), intervalo QT prolongado o alteraciones del complejo QRS.

2) Miocardiopatía: se puede observar hipertrofia, dilatación y disfunción sistólica del ventrículo izquierdo.

- Respiratorias: éstas son muy frecuentes. Constituyen una de las principales causas de muerte prematura. Se afectan los músculos respiratorios, incluido el diafragma, lo cual puede alterar la mecánica ventilatoria.

- Gastrointestinales: debidas a debilidad y miotonía de la musculatura.

1) Disfagia.

2) Retraso del vaciamiento gástrico.

3) Estreñimiento alternante con diarrea.

4) Colelitiasis.

- Oculares: cataratas en casi $100 \%$ de los pacientes o ptosis palpebral.

- Endocrinas y dermatológicas

La gran diversidad de complicaciones que se pueden presentar en estos pacientes en el quirófano y en las unidades de recuperación postanestésicas (URPA) hacen que la conducta anestésica de estos enfermos suponga un reto para el anestesiólogo.

\section{EXPOSICIÓN DEL CASO}

Paciente masculino de 60 años programado para resección transuretral prostática por hiperplasia benigna de próstata. Se administraron $300 \mathrm{mg}$ vía oral de ranitidina la noche previa para disminuir el riesgo de broncoaspiración.

A su llegada al quirófano se revisó la valoración preanestésica donde consta enfermedad de Steinert e implantación de marcapasos por bloqueo AV de tercer grado, que fue revisado previo a la intervención quirúrgica y se informó como normofuncionante por el Servicio de Cardiología. No existen otros antecedentes de interés ni intervenciones quirúrgicas previas.

La exploración física mostró un fenotipo compatible: calvicie frontal y facies característica con sonrisa invertida y mejillas hundidas. No existieron signos que sugirieran la presencia de valvulopatías ni debilidad muscular manifiesta.
Se realizó monitorización estándar. Presión arterial inicial 100/80 mmHg, frecuencia cardíaca 54 latidos por minuto, frecuencia respiratoria 14 respiraciones por minuto y saturación de $\mathrm{O}_{2} 99 \%$. No se realizó monitorización de la temperatura por no disponer de dispositivos cutáneos y evitar el uso de sonda esofágica en paciente consciente, en ausencia de clínica que sugiera hipo- o hipertermia.

Se decidió no utilizar medicación preanestésica en el perioperatorio con benzodiacepinas para evitar una posible depresión respiratoria dada la afectación que estos pacientes pueden tener de la musculatura respiratoria y el aumento de la sensibilidad a los efectos depresores de las mismas. Al tener en cuenta la enfermedad del paciente se optó por anestesia intradural con bupivacaína hiperbárica $0.5 \% 7 \mathrm{mg}$ combinada con fentanilo $10 \mu \mathrm{g}$.

Se administró suero salino fisiológico a $100 \mathrm{~mL} / \mathrm{h}$ para reposición hídrica y recuperación de volumen, así como oxígeno por gafas nasales a $2 \mathrm{~L} / \mathrm{min}$; se aplicó manta térmica. No apareció ninguna complicación durante los 50 minutos de intervención, con un tiempo de resección de 35 minutos.

Tras la intervención el paciente pasó a la URPA donde continuó con monitorización estándar. Tuvo lugar la recuperación completa del bloqueo motor, consiguiéndose una analgesia intravenosa adecuada con paracetamol $1 \mathrm{~g}$ y metamizol 2 g. Dada la buena evolución, se decidió su alta a planta de hospitalización para seguimiento y tratamiento médico, sin presentar ninguna incidencia postoperatoria. Es dado de alta a su casa a las 48 horas sin ningún contratiempo.

\section{DISCUSIÓN $\mathbf{N}^{(7-9)}$}

\section{Período preoperatorio}

La valoración preanestésica es fundamental, se debe comprobar la afectación muscular existente; además, es esencial la valoración de las manifestaciones extramusculares.

Hay que realizar una adecuada evaluación del estatus y funcionalidad ventilatoria, solicitar valoración espirométrica, y polisomnografía si procediese, para comprobar los volúmenes pulmonares dada su frecuente reducción, y eventualmente la optimización por el Servicio de Neumología.

Se debe examinar de forma cautelosa el ECG en busca de alteraciones de la conducción y signos de cardiopatías y remitir al Servicio de Cardiología ante cualquier anormalidad significativa.

No se ha documentado una mayor incidencia de vía aérea difícil en estos pacientes, pero se debe evitar inducir en el intraoperatorio una miotonía que resulte en ventilación o intubación imposible.

Se debe incidir de manera reiterada sobre el ayuno preoperatorio, así como pautar profilaxis para la broncoaspiración (antagonista H2 y/o citrato sódico y/o procinéticos). 
El estrés quirúrgico y las técnicas utilizadas pueden interferir en el curso de la enfermedad, por lo que es muy importante una correcta conducta perioperatoria para no provocar un empeoramiento o aparición de nuevas manifestaciones clínicas de la enfermedad.

\section{Período intraoperatorio}

La regla principal es impedir que se desencadene una crisis miotónica, por ello tendremos que contar con la potencial sensibilidad incrementada de estos pacientes a fármacos anestésicos comúnmente utilizados.

La anestesia locorregional (bloqueos regionales/técnicas neuroaxiales) es utilizada con frecuencia en estos pacientes y comunicada como segura, en la actualidad, ésta es considerada la técnica anestésica de elección. Además, es necesario tener en cuenta que la adición de benzodiacepinas u opioides puede ocasionar problemas ventilatorios y aspiración, por lo que su uso debe ser cauteloso. Habrá que tener especial cuidado al momento de realizar bloqueos regionales guiados por neuroestimulador, dado que las contracciones inducidas pueden ocasionar contracciones miotónicas. Son preferibles las técnicas ecoguiadas.

Agentes hipnóticos intravenosos como propofol o etomidato junto con opioides de acción corta (remifentanilo) son fármacos seguros en la práctica anestésica, por lo que se debe utilizar esta práctica recomendada. Los anestésicos volátiles pueden inducir un efecto secundario grave como es la hipertermia maligna, por lo que hoy en día se recomienda evitar su uso en estos pacientes. Se debe considerar la inducción de secuencia rápida con presión cricoidea para disminuir los riesgos de broncoaspiración.

Hay que tener especial precaución con los bloqueadores neuromusculares y sus antagonistas. Los bloqueadores neuromusculares despolarizantes, como la succinilcolina, están contraindicados porque provocan fasciculaciones y pueden inducir una contracción muscular mantenida con rigidez que llegue a dificultar e incluso imposibilitar la ventilación e intubación de los pacientes. Por otro lado, los bloqueadores neuromusculares no despolarizantes, como el rocuronio, pueden ser utilizados de manera más segura, siempre que se tenga en cuenta que se puede prolongar su tiempo de duración total. A la hora de revertir estos fármacos se prefiere el uso del sugammadex al de la neostigmina, ya que esta última puede precipitar una miotonía por la sensibilidad incrementada del músculo a los efectos estimulantes de la acetilcolina.

Se deben cumplir los requisitos mínimos exigibles de monitorización estándar, y añadir la medición de la temperatura si se prevé una duración de la cirugía superior a 30' o incluso en cirugías más cortas. Si se va a realizar anestesia general se deben al menos añadir la capnografía y la monitorización del bloqueo neuromuscular. La utilización de monitorización adicional será establecida a juicio del anestesiólogo responsable, en función de las necesidades del caso.

Hay que tener en consideración los fluidos que se van a infundir e intentar evitar aquellos con alto contenido en potasio (capacidad reducida de la bomba sodio/potasio). Además, se deben emplear calentadores corporales y de fluidos, ya que el frío y el temblor pueden desencadenar una crisis miotónica. Finalmente, la disposición de un desfibrilador externo cerca del quirófano, así como dantroleno y fenitoína pueden ser útiles en caso de aparición de efectos adversos.

\section{Período postoperatorio}

Las complicaciones que pueden aparecer son consecuencia, fundamentalmente, de las alteraciones ventilatorias y cardíacas. Para disminuir estos riesgos la extubación se debe realizar con el paciente despierto y siempre tras comprobar una recuperación completa del bloqueo neuromuscular (TOF $>0.9$ ) en caso de usarse bloqueadores neuromusculares.

La infiltración de la herida o la realización de técnicas locorregionales, junto con los AINEs y paracetamol, deben elegirse frente a los opioides para el control del dolor postoperatorio. En la URPA se debe proporcionar una buena calidad analgésica para permitir una fisioterapia respiratoria precoz.

Por último, debe procurarse la disponibilidad de ventilación mecánica no invasiva para este período postoperatorio y tener en cuenta que si al paciente se le ha sometido a una anestesia general con bloqueadores neuromusculares se debería extremar la vigilancia durante las primeras 24 horas y haber previsto con el Servicio de Cuidados Intensivos la posibilidad de un ingreso programado o urgente en dicha unidad.

\section{CONCLUSIONES}

- Las principales manifestaciones clínicas de la enfermedad de Steinert son la miotonía y la debilidad muscular, incluidos los músculos respiratorios, junto con trastornos del ritmo cardíaco que se deben valorar en la consulta preanestésica.

- La anestesia locorregional se considera en la actualidad la técnica anestésica de elección.

- Si se tiene que realizar una anestesia general se recomienda propofol o etomidato junto con opioides de acción corta. No se ha documentado mayor incidencia de vía aérea difícil.

- Los bloqueadores neuromusculares despolarizantes están contraindicados.

- Los bloqueadores neuromusculares no despolarizantes pueden utilizarse de forma más segura, pero hay que tener en cuenta que se pude prolongar su tiempo de duración total.

- La reversión de los bloqueadores neuromusculares es preferible con sugammadex que con neostigmina. 
- Las técnicas locorregionales, junto con los AINEs y paracetamol, deben elegirse frente a los opiáceos para el control del dolor postoperatorio.
- Si el paciente ha sido sometido a una anestesia general con relajación neuromuscular se debería extremar la vigilancia durante las primeras 24 horas.

\section{REFERENCIAS}

1. Thornton CA. Myotonic dystrophy. Neurol Clin. 2014;32:705-719. Available in: http://doi.org/10.1016/j.ncl.2014.04.011

2. Bird TD. Myotonic dystrophy type 1. In GeneReviews ${ }^{\circledR}$ [Internet]: University of Washington, Seattle; 2018. Available in: https://www. ncbi.nlm.nih.gov/books/NBK1165/

3. Gutiérrez GG, Díaz-Manera J, Almendrote M, Azriel S, Bárcena JE, García PC, et al. Guía clínica para el diagnóstico y seguimiento de la distrofia miotónica tipo 1, DM1 o enfermedad de Steinert. Med Clin (Barc). 2019;153:82.e1-82.e17. Disponible en: http://doi.org/10.1016/j. medcli.2018.10.028

4. Pelargonio G, Russo AD, Sanna T, De Martino G, Bellocci F. Myotonic dystrophy and the heart. Heart. 2002;88:665-670. Available in: http:// dx.doi.org/10.1136/heart.88.6.665

5. Bellini M, Biagi S, Stasi C, Costa F, Mumolo MG, Ricchiuti A, et al. Gastrointestinal manifestations in myotonic muscular dystrophy. World J Gastroenterol. 2006;12:1821. Available in: https://doi.org/10.3748/wjg. v12.i12.1821
6. Hawkins AM, Hawkins CL, Abdul Razak K, Khoo TK, Tran K, Jackson RV. Respiratory dysfunction in myotonic dystrophy type 1: A systematic review. Neuromuscul Disord. 2019;29:198-212. Available in: https://doi. org/10.1016/j.nmd.2018.12.002

7. Kerstin H. Recomendaciones para anestesia en pacientes que afectados por distrofia miotónica 1 y 2. Enciclopedia de Orphanet. 2014 [13/03/2019]. Disponible en: https://www.orpha.net/data/patho/Pro/es/ Distrofia-miotonica-1-y-2_ES.pdf.

8. Oriol-López S, Hernández-Bernal C. Anestesia en la distrofia muscular de Steinert. Rev Mex Anest. 2010;33:160-165.

9. Catena V, Del Monte DD, Rubini A, Guccione C, Ricagna F, Gangeri G, et al. Anesthesia and myotonic dystrophy (Steinert's syndrome). The role of total intravenous anesthesia with propofol, cisatracurium and remifentanyl. Case report. Minerva Anestesiol. 2007;73:475-479. 